Piet Jongbloet, Radboud University Nijmegen Medical Centre, Nijmegen, The Netherlands. Email: p.jongbloet@skynet.be

doi: 10.1192/bjp.192.4.313a

Authors' reply: Jongbloet provides an alternative explanation of our findings about the effect of month of birth on suicide that is based on the oocyte origins hypothesis as opposed to the maternal-foetal origin hypothesis. The oocyte hypothesis (also referred to in literature as 'conception hypothesis') may have significant implications in psychiatry. The intricate interplay between non-optimal oocyte maturation and genes results in a complex pathogenesis of the resultant foetuses or individuals. This occurs in well-timed menstrual cycles, but more so in instances of distorted hormonal tuning, not only in deprived socio-economic conditions but also at the extremes of maternal reproductive life, among endocrinologically unbalanced mothers, after very short pregnancy intervals during the seasonal transitions of the 'ovulatory' seasons, etc. ${ }^{1}$ A similar broad spectrum of male-biased developmental anomalies - low birth weight and length, small stature at school age or adulthood, morbidity, and mortality is present in all these circumstances. ${ }^{2}$

To illustrate the oocyte or conception hypothesis in practical terms: mothers with low socio-economic status are known to suffer from more menstrual disorders, ${ }^{3}$ low standards of nutrition and abnormal body mass index. They also are more likely to be smokers or to misuse drugs ${ }^{4}$ and to employ less safe methods of contraception resulting in unplanned and unwanted pregnancies, particularly at the extremes of maternal reproductive age and during the postpartum restoration of the ovulatory pattern (i.e. after very short inter-pregnancy intervals). They are likely to have non-optimal oocyte maturation, thus rendering the offspring vulnerable to low birth weight and certain psychiatric disorders. However, we are not clear as to how this hypothesis actually differs from the maternal-foetal origin hypothesis used to explain our findings. ${ }^{5}$

The geographical latitude effect in incidence rates of suicide in England, Wales and elsewhere is assumed by Jongbloet to be a consequence of the stronger seasonal ovulatory pattern the further away from the equator, just as in animals, and, in turn, stronger transitional stages between the ovulatory seasons and, thus, more poor-quality oocytes. However, the only way to accept or reject this concept is by demonstrating the same increase of suicide incidence rate - and of other disease entities or behaviour of complex origin.

We are also grateful to Chotai for his comments. Although we did not look at hanging in relation to month of birth in our study, we did in fact examine the relationship between month of birth and violent suicide (including hanging) as opposed no nonviolent suicide, but found no significant association. However, a previous study, ${ }^{6}$ in an attempt to replicate the findings of Chotai et $a l^{7}$ showed that those born during the season January-April were more likely to prefer hanging than poisoning: data from North Cheshire $(n=502)$ appeared to suggest that suicide by hanging was significantly more frequent in those born in the summer months compared with those who used other methods such as poisoning by solids or gases. The findings were not in keeping with reports by Chotai et al. However, methodological limitations of the North Cheshire study, including a relatively small sample size, have significantly limited its inferential value. Studies with sufficient power to detect the association between month of birth and risk of hanging are required to show whether one truly exists. Seasonality of birth studies in relation to suicide may enhance our understanding of some biological aspects in the aetiology of suicide such as the oocyte origins hypothesis proposed by Jongbloet.

1 Jongbloet PH, Zielhuis GA, Groenewoud HMM, Pasker-de Jong PCM. The secular trends in male: female ratio at birth in postwar industrialized countries. Env Health Perspect 2001; 109: 749-52.

2 Jongbloet $\mathrm{PH}$. The ageing of gamete in relation to birth control failures and Down syndrome. Eur J Pediatr 1985; 144: 343-7.

3 Power $\mathrm{C}$, Matthews S. Origins of health inequalities in a national population sample. Lancet 1997; 350: 1584-9.

4 Pierce JP, Fiore MC, Novotny TE, Hatziandreu EJ, Davis RM. Trends in cigarette smoking in the United States. JAMA 1989; 61: 56-60.

5 Salib E, Cortina-Borja M. Effect of month of birth on the risk of suicide. $\mathrm{Br} J$ Psychiatry 2006; 188: 416-22.

6 Salib E. Month of birth and suicide: an exploratory study. Int J Psychiatr Clin Pract 2001; 6: 39-44.

7 Chotai J, Salander Renberg E, Jacobsson L. Season of birth associated with the age and method of suicide. Arch Suicide Res 1999; 5: 245-54.

Emad Salib, Liverpool University, Hollins Park Hospital, Warrington WA2 8WA Email: esalib@hotmail.com; Mario Cortina-Borja, Centre for Paediatric Epidemiology and Biostatistics, Institute of Child Health, University College London, UK

doi: 10.1192/bjp.192.4.314

\section{Reattribution for medically unexplained symptoms}

Morriss et al $^{1}$ performed a high-quality cluster randomised controlled trial in which reattribution for medically unexplained symptoms was taught to general practitioners (GPs). We compliment the authors on this trial. Strong points of their trial are the avoidance of selection bias by using an independent GP for inclusion, and the inclusion of patients for whom unexplained symptoms of sufficient duration were the reason for the encounter. However, we have some critical comments as well.

First, the training of GPs took only 6 hours and was performed by non-expert trainers. Reattribution is not an easy technique to learn. Other researchers have used training programmes of at least 20 hours. ${ }^{2,3}$ The trainers in this study were three nurses and a psychologist. Although they were prepared intensively, they might not have been familiar enough with GP consultations. Consequently, we have doubts about the thoroughness and effectiveness of the training for GPs.

Second, the effect of reattribution training on doctor-patient communication has been evaluated in only one consultation. Reattribution usually takes more than one consultation. ${ }^{4}$ Making an inventory of the problems and broadening the agenda can lead to quite a disturbance of the normal flow of the consultation. Patients often need more time to make a link between their psychosocial and physical problems. In the article it seems like it was mostly the doctor who made the link. This does not fit into the original reattribution model. A negotiating style is needed in order to let the patient raise the possibility of a link him- or herself. ${ }^{4}$ For the purpose of effective reattribution, the patient has to come up with the link and not the doctor. ${ }^{2,3}$

Third, we know that the effectiveness of psychological treatments consists of specific and non-specific effects. Non-specific effects are effects caused by mutual trust, empathy and shared understanding. ${ }^{5}$ The training in reattribution and applying it might have influenced the physicians' relation with the patient negatively because of the physicians being absorbed by the application of the new intervention. Less attention for empathy and other non-specific effects might have been an additional cause for the absence of treatment effects.

Finally, it is a pity that the authors did not differentiate the outcome effects for subgroups. Patients with medically unexplained symptoms form a heterogeneous group. 'Treatment effects 